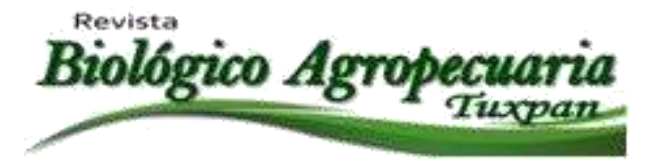

\title{
Caracterización del estado nutricional foliar para níquel en nogal pecanero
}

Characterization of foliar nutritional status for nickel in pecan

\author{
Alanís Fierro Ana Karen ${ }^{\bowtie}$, Juan Manuel Soto-Parra, Esteban Sánchez-Chávez, Rosa María Yáñez- \\ Muñoz y Francisco Javier Piña-Ramírez
}

Facultad de Ciencias Agrotecnológicas. Universidad Autónoma de Chihuahua. Teléfono: (614)2412687 Dirección: campus universitario s/n C.P. 31310 A.P. 24, Chihuahua, Chihuaua.

${ }^{\circledR}$ Autor para correspondencia: karenalanis@live.com.mx

Recibido: $11 / 01 / 2014$
Aceptado: 14/07/2014

\section{RESUMEN}

Chihuahua es el principal productor de nuez en México con 58 mil 810 ha plantadas y una producción para el 2011 de 71 mil 689 T que representó el 80\% del total nacional. El níquel elemento que pasó de ser toxico a esencial, es requerido por la planta para un buen desarrollo y producción. Se caracterizó el comportamiento nutricional foliar de níquel en nogal en los principales municipios productores. Se muestrearon 68 huertos y 399 árboles 'Western Schley', se generó el estándar de referencia mediante la metodología del Diagnóstico Diferencial Integrado (DDI). El estándar generado, coincidió con lo reportado. Aldama presentó un $60 \%$ suficiente y $40 \%$ deficiente; región Delicias $57 \%$ fue suficiente y $43 \%$ deficiente; Camargo se ubicó en rango suficiente con $86 \%$ y $14 \%$ bajo; Jiménez fue suficiente $78 \%$ y $18 \% \%$ alto. Del universo muestreado $74 \%$ estuvo en rango suficiente, $16 \%$ bajo y un $10 \%$ alto.

Palabras clave: Carya illinoienensis (Wangenh.) K Koch) 'Western Schley', estándar nutricional, Diagnóstico Diferencial Integrado (DDI), frecuencias.

\begin{abstract}
Chihuahua is the largest producer pecan in Mexico with 58,810 ha planted and production for 2011 of $71,689 \mathrm{~T}$ represented $80 \%$ of the national total. The nickel element that went from being toxic to essential is required by the plant for good growth and production. It is characterized the foliar nutrition behavior of nickel in the main towns pecan producers. 68 orchards were sampled and 399 'Western Schley' trees, generated by the reference standard methodology of Integrated Differential Diagnosis (IDD). The generated standard, agreed with those reported. Aldama presented sufficient $60 \%$ and $40 \%$
\end{abstract}

Revista Científica Biológico Agropecuaria Tuxpan 2 (1) 
poor, 57\%; Delicias Region was 57\% sufficient and $43 \%$ poor; Camargo was at sufficient range to $86 \%$ and $14 \%$ under, Jimenez was sufficient $78 \%$ and $18 \%$ high. $74 \%$ of the universe was sampled in sufficient, $16 \%$ low and $10 \%$ high.

Keywords: Carya illinoienensis (Wangenh.) K Koch) 'Western Schley', nutritional standard, Integrated Differential Diagnosis (IDD), frequencies.

\section{INTRODUCCION}

Chihuahua es el principal productor de nuez en México con 58 mil 810 ha plantadas y una producción para el 2011 de 71 mil 689 T que representó el $80 \%$ del total nacional (SIAP, 2011). En la actualidad se tiene conocimiento de que el nogal requiere de 17 elementos esenciales para su buen desarrollo entre estos esta reportado el Níquel (Ni). El Níquel como lo citan algunos autores es esencial para la nutrición de las plantas (Brown et al. 1987) ya que es un componente de la enzima ureasa (Dixon et al., 2004). Se sabe relativamente poco sobre el papel del Níquel en la nutrición vegetal, fisiológica y el metabolismo especialmente en las especies leñosas perennes como Pecan (Vadez, 2000). Un síntoma clave de deficiencia es enanismo de las hojas y foliolos (Wood et al, 2004) "Oreja de ratón" es la expresión utilizada para describir síntomas peculiares observados en las hojas del pecano (Carya illinoinensis) y en ciertas otras plantas. La punta de las hojas jóvenes afectadas presenta, anchas oscuras y redondeada, pareciéndose a las hojas de ratón.

Uvalle-Bueno (1993) recomienda que los fenómenos que afectan a las plantas deben estudiarse en una forma holística en el contexto de la relación agua-suelo-planta-atmósfera, debido a que las plantas ganan y pierden nutrimentos continuamente, y la toma neta cambia con el tiempo y difiere entre nutrimentos. La utilización de los valores de referencia del Diagnostico Diferencial Integrado (DDI) para derivar intervalos nutricionales permite llevar a cabo el análisis vegetal con propósitos de monitoreo, predicción y los índices nutricionales del DDI entre plantas sanas y enfermas otorgan la posibilidad de comprender la naturaleza nutricional o fisiológica del desbalance metabólico. El presente trabajo tiene como objetivo caracterizar el comportamiento de níquel en nogal pecanero en los principales municipios productores del estado de Chihuahua, para detectar necesidades del nutriente que deriven en medidas preventivas y correctivas para mejorar nutrición y elevar producción.

\section{MATERIALES Y MÉTODOS}

El estudio se realizó en el municipio de Camargo, Aldama, Jiménez, Meoqui, Saucillo y Delicias del estado de Chihuahua. Se seleccionaron 68 huertos representativos de los municipios los cuales se jerarquizaron de acuerdo al esquema de Diagnóstico Diferencial Integrado. Dentro de cada huerto se seleccionaron de 4 a 36 árboles distribuidos lo más posible en un área representativa de cada uno de los huertos, "sanos nutricionalmente". En cada uno de los árboles sanos que se seleccionaron, se tomaron muestras de hojas.

\section{Determinación Nutricional Foliar}

Níquel (Ni) (Mezcla Digestora Triácida y Espectrofotometría de Absorción Atómica).

Revista Científica Biológico Agropecuaria Tuxpan 2 (1)

ISSN: 2007-6940 
Generación de Estándares Nutricionales Foliares

Con los resultados de los análisis foliares se obtuvieron para níquel nueve niveles, valores o concentraciones nutricionales: Deficiente (D), Muy bajo (mB), Bajo (B), Medianamente bajo (MB), Suficiente(S), Medianamente alto(MA), Alto (A), Muy alto (mA) y Exceso (E); definidos como los nueve posibles rangos en los que puede estar el níquel en una planta, relacionados con el crecimiento relativo o la producción del cultivo (Uvalle-Bueno, 1993).

\section{RESULTADOS Y DISCUSIÓN}

\section{Generación de estándares nutricionales foliares}

Los estándares nutricionales generados mediante Diagnóstico Diferencial Integrado se muestran en el Cuadro 1 en donde cada una de las categorías del contenido nutricional lleva asociado el valor crítico DDI y a partir de la media se van generando los valores correspondientes derivando en el intervalo respectivo.

De esta manera, se obtienen los nueve rangos nutricionales lo que permite identificar con mayor detalle el estado nutrimental así como la presencia de desbalances nutricionales y / o fisiológicos de la planta.

\section{Comparación con lo Reportado}

Es importante mencionar algunos aspectos relacionados con los valores de referencia reportados por algunos. Generalmente no se menciona como llegaron a obtener los valores que reportaron, que técnicas de muestreo de campo para la recolección de muestras siguieron, determinaciones analíticas de laboratorio y sustento para el procesamiento de la información, de tal manera que ya no solo se está extrapolando situaciones peculiares del contexto agua- suelo- planta-clima, sino también técnicas analíticas que pueden ser no apropiadas bajo ciertas situaciones .Autor consultado se muestra en el Cuadro 1.

Cuadro 1. Rangos nutricionales para níquel en hojas de nogal pecanero.

\begin{tabular}{cccc}
\hline Nutriente & Bajo & Normal & alto \\
\hline Níquel & $2.8-8.4$ & $8.5-14.2$ & $14.3-20$ \\
\hline $\begin{array}{l}\text { Andrew et al., } \\
2006\end{array}$ & & & \\
\hline
\end{tabular}

\section{Frecuencias observadas por huertos}

En el rango obtenido para el municipio de Camargo podemos observar en la figura 1 que está bien, ya que la mayoría tiene un $94 \%$ que entra en suficiente, bajo el mínimo de $6 \%$ y ninguno entra en alto. 


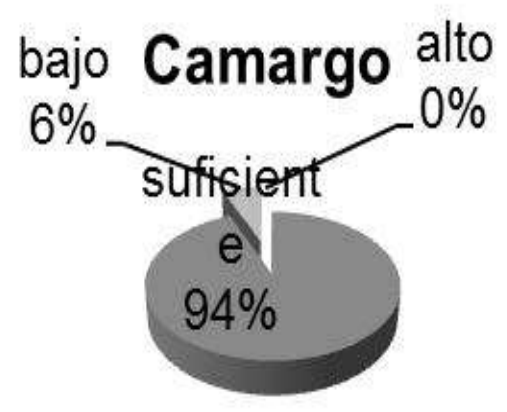

Figura 1. Total de frecuencias observadas a nivel huerto en el municipio de Camargo.

Para el municipio de Aldama a nivel huerto podemos ver que entra en un buen rango ya que también tiene suficiencia en su mayoría, o hacer aplicaciones en bajas dosis (Fig. 2).

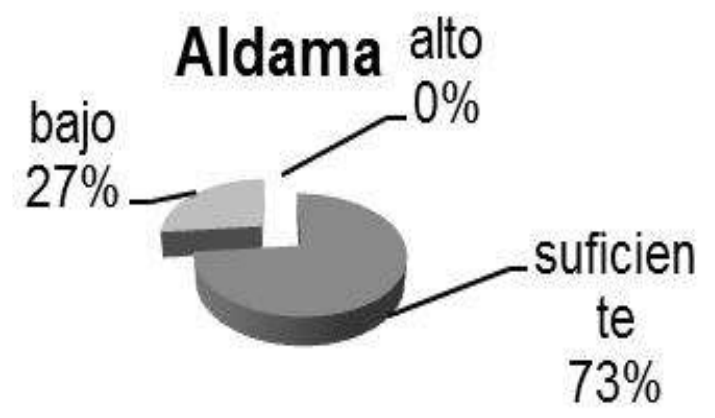

Figura 2. Total de frecuencias observadas a nivel huerto en el municipio de Aldama.

En el municipio de Delicias se ve un problema e deficiencia de níquel (Fig. 3).

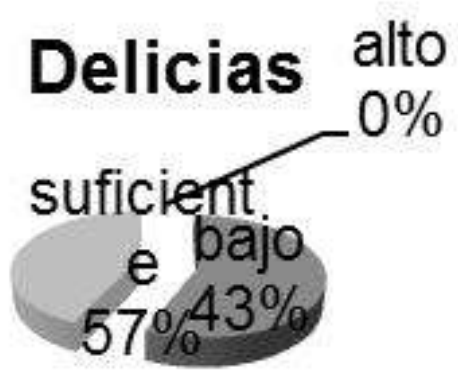

Figura 3. Total de frecuencias observadas a nivel huerto en el municipio de Delicias. 
En el municipio de Jiménez, como observamos en la figura 4 podemos observar que un $20 \%$ que corresponde a un nivel alto es representativo para tomar medidas.

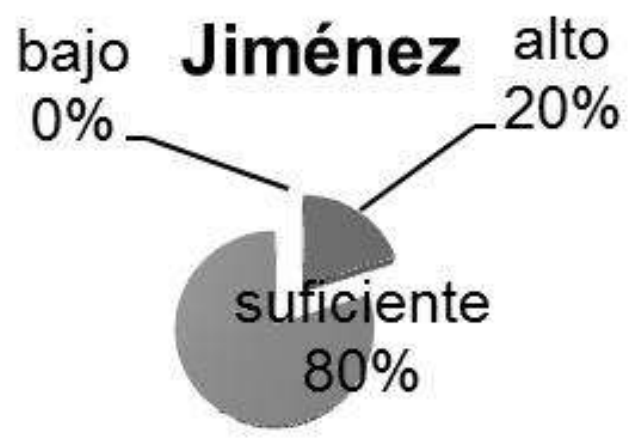

Figura 4. Total de frecuencias observadas a nivel huerto en el municipio de Jiménez.

\section{CONCLUSIONES}

El estándar foliar para níquel que obtuvimos fue apropiado, porque coincide con lo reportado. En el total de arboles analizados, se registro que en su mayoría con un total de 238 árboles están en el nivel de suficiente, mientras que 53 árboles están bajos y 32 altos del estándar encontrado. En el total a nivel huerto, el $23 \%$ cayó en bajo mientras que $3 \%$ fue alto y un $74 \%$ en suficiente, lo que indica que en general está en un buen nivel. Para poder profundizar más este tema, se puede hacer también análisis no solo foliar sino también de suelos, también se recomienda que se haga un examen de enzimas en específico de ureasa, ya que sabemos que el níquel es un compuesto de la enzima.

\section{LITERATURA CITADA}

Andrew, P. P. y James, L. W. 2006. Leaf Nutrient Levels for Pecans.

Brown, P. H., Welch, R. M. y Cary, E .E. 1987. Nickel micronutrient nessential for all higher plants. Plant Physiol. 85, 801

Dixon, N. E., Blakely, R. L. y Zerner, B. 2004. Jack-bean ureas the involvement of active site nickel in inhibition by mercaptoethanol and phosphoramidate, and pluoride. Can. Biochem. 481 p. https://doi.org/10.1139/080-064

SIAP-SAGARPA. 2011, Resumen de cierre y cosechas para cultivos perenes, sub delegación de planeación. SAGARPA. Delegación estatal chihuahua, México.

Uvalle-Bueno, J. X. 1993. Curso de Nutrición Vegetal enfocado al Diagnóstico Diferencial Integrado (DDI), impartido del 8 al 12 de noviembre de 1993. Facultad de Fruticultura, Universidad Autónoma de Chihuahua. Chihuahua, Chih. México. 40 p. https://doi.org/10.24327/ijrsr.2017.0803.0060

Valdez, V. y Sinclair, T. R. 2000. Ureide degradation pathways in intact soybean leaves. J Exp Bot. 51: 1459-1465 https://doi.org/10.1093/jxb/51.349.1459 
Wood, B. W., Reilly, C. C., Nyczepir, A.P.

2004. Mouse-ear of pecan:

Symptomology and occurrence.

HortScience, 38: 87-94.

https://doi.org/10.21273/hortsci.39.1.87

Copyright (c) 2014 Ana Karen Alanis Fierro, JuanMarwel Soto Parra, Esteb an Sánchez Chávez, Rosa Maria Yáñez Muñoz yFrancisco Javier Piña Ramirez

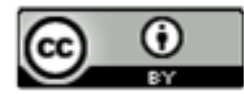

Este texto está protegido por una licencia Creative Commons 4.0

Usted es libre para Compartir —copiar y redistribuir el material en cualquier medio of ormato-y Ad aptar el documento —remezclar, transformar y crear a partir del material- para cualquier propósito, inchso para fines comerciales, siempre que cumpla la condición de:

Atribución: Usted debe dar crédito a la obra original de manera adecuada, proporcionar un enlace a la licencia, e indicar si se han realizado cambios. Puede hacerlo en cualquier forma razonable, pero no de forma tal que sugiera que tiene el apoyo del licenciante olo recibe por el usoque hace de la obra.

Resumendelicencia - Textocompletodelalicerria 